\title{
A MORE SIMPLE ALTERNATIVE FOR THE SOLUTION OF THE SECOND ORDER DESIGN PROBLEM OF GEODETIC NETWORKS
}

\author{
Kamal A. Atia ${ }^{*}$, Abo El-Hassan M. Rahil ${ }^{* *}$ and Ahmad F.Abo- Gohnim ${ }^{* * *}$ \\ * Civil Engineering Dep,,Faculty of Eng., Alexandria University, Egypt. \\ ** Civil Engineering Dep.,Faculty of Eng., Minufiya University, Egypt. \\ *** Civil Engineer
}

\begin{abstract}
A more simple alternative for the solution of the second order design problem of geodetic networks with respect to realization of the results has been developed. One of the main poblemes, which can arise when solving the SOD problem is that, the resulting weight vector may not reproduce a cofactor matrix, which ensures the criteria requerements, in some parts of the network, or all over the network. In this paper, this problem is treated by modifying the resulting weight vector linearly, by its multiplication by a scaler value . Numerical examples are given to illustrate its application. The commercial "Matlab" program was used to solve the case studies.
\end{abstract}

\section{INTRODUCTION}

The optimization of the design of the geodetic networks, is accepted to be classified to four design problem (orders) namely; Zero-Order Design (ZOD), First Order Design (FOD), Second Order Design (SOD), and Third Order Design (THOD), ZOD is the problem of definding the datum or coordinate system, FOD is the problem of constructing the optimum shape or configuration of the network, SOD is the problem of determining the optimum weight of the observed quantities and finally THOD is the problem of improving the quality of existing networks. The second-order design (SOD) problem is defined as, the search for the optimal observationl weights, of a given observational plan, under a given criteria, which is usually formed as an ideal criterion matrix. The resulting weight vector may not reproduce a covariance matrix, which ensures the required criteria in some elements. So, in this study, the resulting weight vector can be linearly modified, by its multiplication by a suitable scaler to ensure the required criteria all over the network.

Manuscript received from ENG .AHMAD F.ABO-GOHNIM on : $21 / 3 / 2000$ Accepted on : 28/3/2000 Engineering Research Bulletin, Vol 23,No 2, 2000 Minufiya University, Faculty of Engineering, Shebien EI-Kom, Egypt, ISSN 1110-1180 
This modification is done depending on the comparison between the resulting criteria and the adopted ones, and then, multiplying the resulting weight vector by this scaler.The simple least - squares solution, by approximating an inverse criterion matrix, is applied to the case - studies in the present investigation. The unit matrix is used successfully as a criterion matrix in all solved examples. The free network concept is used in these examples, which is the suitable one for obtaining the properties of homogeneity and isotropy. A more simple alternative for the solution of the second order design problem of geodetic networks with respect to realization of the results has been developed.

\section{THE GENERAL CONCEPT OF THE SOLUTON OF THE SOD PROBLEM OF GEODETIC NETWORKS}

The general concept of the solution of the SOD problem involves three steps ( Schmitt, 1985):

1 - A pure weight optimization with respect to the criterion matrix;

2- The optimum weights ( resulted from stepl) are approximated by a detailed plan of instruments, procedures and repetiton numbers of the observations.

3- This design is analyzed; whether it fits the network requirements. Thus, when substituting by the resulting weight matrix $P$, the criteria adopted and the requirements, are obtained or not. One can see that, whether the resulting weight vector $P$, is modified, until the criterion are satisfied.

\subsection{FORMULATION OF THE ADOPTED CRITERIA}

There are two different methods to express the required criteria, which are adopted, when solving the SOD optimization problems. The first minimizes or maximizes a scalar risk function, for example, the trace of the cofactor matrix $Q_{x}$ : trace $\left(Q_{x}\right)=\min$. The second type of the adopted criteria are the so- called criterion matrices. The determination of the criterion matrix is considered as a central problem, when solving the SOD problems. Generally, the geodetic networks are said to be optimum, if it has the properties of homogeneity and isotropy. The simplest cofactor matrix which leads to these proberties, is the unit matrix, in which the diagonal elementsare equally sized, and the off- diagonal elements are zeros. The unit matrix can be considered as a special case of the Taylor-Karmen structure criterion matrices, if zero correlation functions are introduced.

\subsection{SOLUTON METHODS OF THE SECOND - ORDER DESIGN PROBLEM}

All the strategies of the solution of the optimization problems, and especially the second-order design problems, are considered as one two categories:

Computer simulation and analytical methods. In the analytical methods, two basic approaches are used in the solution. The first is based on the concept of best - fitting the user precision, in a least - squares sense. The 
other uses the techniques from the operation research called mathematical programming. In this study, we will deal with the least squares solution.

\subsubsection{THE GENERALIZED MATRIX APPROACHE ( THE SIMPLE SOLUTIONS)}

These approaches are called the simple approaches, since it depends on finding the the optimal weights, from the simple relation $\left(\mathrm{A}^{\mathrm{T}} \mathrm{PA}\right)^{+}=\mathrm{Q}_{\mathrm{x}}$, i.e through one relation only. ( where: ()$^{+}$is used to include either the constrained and free networks, and regular or singular criterion matrices, as well ). The solution is obtained by formulating a redundant system of equations in the unknown weight vector $\mathrm{P}$, to be solved by the least squares principle, or equivalently, by using the generalized inverse of matrices, especially the Moore Penrose g-inverse.The solution of a redundant system of equations using the Moore Penrose g-inverse ( ${ }^{+}$, will yield the same results as applying the least-squares principle. The two approaches yield a solution vector with a minimum norm and also, the residual vector with a minimum norm. The optimaity of the simple leastsquares (or the pseudo-inverse approach) is achieved depending on the fact that, the resultig solution vector $p$, will have a minimum norm $\left(p^{T} p=\min\right.$.) ( Schaffrin, 1981). This property of the solution vector $p$ indicates the minimum cost, since the weights are related inversely to the variances. So, the minimization of the weights indicates the larger the required variances, respectively, less precise instruments, the simpler the procedures and less repetition numbers of the observations. The simple least-squares approaches can be classified into three kinds, namely:

1-The direct approaches: which approximate directly the criterion matrix or its inverse.

2-The canonical design: which uses the concept of the sigular value decomposition of the inverse criterion matrix.

3-The iterative approach: which starts by an inital weight vector $\mathrm{p}^{0}$, then, it is updated through an iterative manner, until reaching to the desired one.

In this study, for finding the optimal weights, from the simple relation $\left(\mathrm{A}^{\mathrm{T}} \mathrm{PA}\right)^{+}=\mathrm{Q}_{\mathrm{x}}$ the direct approximation of the inverse criterion matrix have been used as follows: Starting by the cofactor matrix equation :

$$
\mathrm{A}^{\mathrm{T}} \mathrm{PA}=\left(\mathrm{Q}_{\mathrm{rx}}\right)^{+}
$$

Using the Khatri-Rao product, the system (2) can be converted to a system of linear equations for the vector $p$, thus :

with $: p=v e c p$ and

$$
\left(A^{T} \Theta A^{T}\right) p=q
$$

$$
\mathrm{q}=\operatorname{vec} \mathrm{Q}_{\mathrm{rx}}^{+}=\operatorname{vec} \mathrm{p}_{\mathrm{x}}
$$

The solution of Equaion (3) is obtained by (e.g. Schmitt $1978,1979,1985$ )

$$
\mathrm{p}=\left(\mathrm{A}^{\mathrm{T}} \Theta \mathrm{A}^{\mathrm{T}}\right)^{+} \mathrm{q}
$$




\section{A MORE SIMPLE ALTERNATIVE FOR THE SOLUTION OF THE SECOND ORDER DESIGN PROBLEM OF GEODETIC NETWORKS}

In this study, A more simple alternative for the solution of the second order design problem of geodetic networks is introduced. It is generally, based on the least-squares principle, applied to solve the over-determined system of equations in the unknown vector p. Most of the solutions are depending mainly on the precision criteria, expressed by the criterion matrices. Some times, after obtaining the required weight vector, the reproduced covariance matrix, obtained by substituting the resulted weight vector, may not ensure the required criteria in some parts of the network or all over the network. For example, the resulted semi-major axes of the absolute errors ellipses, of some of the net points, may be worse than the adopted ones. This is because these solutions are depending on the least-squares principle, or best-fitting the adopted criterion matrix, and this leads to that, the resulted criteria will be better in some parts and bad in the others. The resulted weight vector will be called the preliminary solution, to be modified-if necessary- to another one, to ensure the required criteria all over the network. This concept can be applied also, if the resulted criteria are greatly better than the adopted ones, yielding an error situation more precise than the adopted one. This will increase the cost and the network will be not economical, for some purposes. Usually, the modified of the preliminary resulted weight vector, will be a linear modification. This means that, the preliminary weight vector will be multiplied by a scaler value, yielding a new vector, which will satisfy the requirements. The preliminary obtained weight vector will be denoted by $\mathrm{p}_{1}$ and the modified one will be denoted by $\mathrm{p}_{2}$. The covariance matrix resulted from the vector $p_{1}$ will be denoted by $\sum_{x 1}$ the modified one by $\sum_{x 2}$. Considering the covariance matrix equation :

$$
\sum_{x 1}=\sigma_{0}^{2}\left(A^{T} P A\right)^{+}
$$

When the weight vector $\left(p_{1}\right)$ is multiplied by a factor $(r)$, it will be changed to say $\left(p_{2}\right)$, such that : $\left(p_{2}=r \cdot p_{1}\right)$

The modified covariance matrix $\sum_{x 2}$ will be :

$$
\begin{aligned}
& \sum_{x 2}=\sigma_{0}^{2}\left(A^{T} P_{2} A\right)^{+}=\sigma_{0}^{2}\left(A^{T} r_{P_{1}} A\right)^{+} \\
& \sum_{x 2}=\frac{1}{r}\left[\sigma_{0}^{2}\left(A^{T} P A\right)^{+}\right]=\frac{1}{r} \sum_{x 1}
\end{aligned}
$$

Thus, any linear transformation to the weight vector $\left(p_{1}\right)$ by a scaler $(r)$ will reduce the elements of the covariance matrix by $\left(\frac{1}{r}\right)$. All the elements of the new covariance matrix $\sum_{x_{2}}$ will equal to the corresponding elements of $\sum_{x 1}$, divided by (r). Schmitt (1985) gave a procedure, to overcome the problem of exisiting any difference between the actual variance-covariance matrix, resulted from the optimal weights $\mathrm{p}_{1}$, and the desired criterion matrix. This is by multiplying the resulted weight vector $p_{1}$ by a factor $\lambda$, such that, the square sum of the residuals $d^{T} d$, will be minimum, where the vector $d$ is the difference between the resulted covariance matrix and the criterion one, computed as follows: 


$$
D=\left(A^{T} p_{1} A\right)^{+}-C_{x}
$$

$d=\operatorname{vec}(D)$. then: $d^{T} d \min$, for $p_{2}=\operatorname{diag}\left(\lambda . p_{1}\right)$ where $\lambda$ can be computed by the operator " trace" as:

$$
\lambda=\frac{\text { trace }\left[\left(A^{T} p_{1} A\right)^{+}\left(A^{T} p_{1} A\right)^{+}\right]}{\text {trace }\left[\left(A^{T} p_{1} A\right)^{+} C_{x}\right]}
$$

This modification may also not ensure that the resulted criteria will satisfy the adopted one, since it considers all the elements of the covariance matrix, to be closer to the corresponding adopted ones, and not consider specific criteria, such as error ellipses.

For this reason, another two methods for the modification of the weight vector ( or the reprouced covariance matrix), are proposed by the authers. The two methods can be summarized as follows:

1- The first method depend on, the comparison between the elements, which express the specific precision criteria (as the major axis of the error ellipse), with the corresponding ones adopted through the criterion matrix. Then, the preliminary weight vector is multiplied by a suitable scaler, which is determined depending on this comparison.

2- The other method uses an iterative modification of the resulted weights, or the repetition numbers of the observations, or some of them. Thus, after obtaining the preliminary solution $\mathrm{p}_{1}$ a realization is done by the available instrument, and the repetition numbers are determined.Then, the actual criteria are computed and compare to the specifications. If there is any weak in any part of the network, the weights of some of the observations, which affect the weak parts, can be achieved through the increasing of the corresponding repetition numbers.

Returning to the first method, it can be achieved by computing the ratios between specific elements of criteria, with the adopted ones in the criterion matrix ( as the major-axes of the absolute error ellipses of all points ). Then, the weight vector is multiplied by the largest value of these ratios. This will ensure that, at least, the largest value of the elements represnting the criteria, will equal the adopted one.

In our discussion, the absolute error ellipses in two-dimensional networks, and the standard deviation of the heights in levelling networks, will be considered, when illustrating the modification methods.

\section{USING THE ABSOLUTE ERROR ELLIPSES FOR THE SOLUTION OF THE SOD PROBLEM :}

The absolute error ellipse is defined by its elements, which are the semi-major axis, the semi-minor axis, and the oriention of them. The discussion will concentrated mainly on the semi-major axis, since it is the worst criteria.

- First, one computes the semi-major axis of the absolute error ellipse $\left(a_{i}\right)$ for any point (I).

- The corresponding semi-major axes, are computed using the criterion matrix and denoted by $\left(\mathrm{a}_{\mathrm{i} 0}\right)$. 
- Compute the ratios $\left(r_{i}=\frac{\overrightarrow{a_{i}}}{a_{i o}^{2}}\right)$ for all points. The largest one

$\left(r i_{\max }\right)$ will be the factor of modification. The new weight vector will be :

$$
\mathrm{p}_{2}=\mathrm{ri}_{\max } \cdot \mathrm{p}_{1}
$$

- The elements of the reproduced covariance matrix will be changed by $\frac{1}{r i_{\max }}$ and the squares of the semi-major axes, of all points will also be reduced by the same value. This is because, the square of the semi-major axis is a linear function of some of the elements of the covariance matrix, without any constant terms. So, any change to the covariance matrix by any scaler will change the square of the semi-major axes by the same value. For example, the semi-major axis $a_{i}$ is given from :

$$
a_{i}^{2}=\frac{1}{2}\left(\sigma_{x i}^{2}+\sigma_{y i}^{2}\right)^{2}+\left(\left(\sigma_{x i}^{2}-\sigma_{y i}^{2}\right)^{2}+4\left(\sigma_{x j i}\right)^{2}\right)^{1 / 2}
$$

It is clear from this equation that, the square of the semi-major axis is a

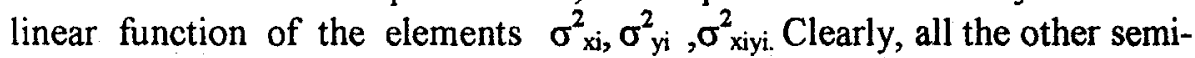
major axes will be reduced by the same value, and all of them will be smaller than the adopted ones. It is to be mentioned here that, this method will success especially when the values of the ratios $\left(r_{i}\right)$ are relatively close to each other, and there is no great difference between them. If there is a great difference, the largest semi-major axis will equal to the criterion one, but the initially small axes will be reduced by the same ratio, and will be more smaller without any need to this high precison, which give high cost. So, the second method can be used in case of existing great difference between these ratios, by increasing only the weights of some observations which affect the weak parts. A similar discussion can be drawn, in case of considering the relative error ellipses, as the studied criteria.

\section{USING THE STANDARD DEVIATIONS FOR THE SOLUTION OF THE SOD PROBLEM :}

The most used criteria for levelling networks, is the standard deviations of the heights of the stations, extracted from the matrix :

$$
\sum_{h}=\left(\begin{array}{cccc}
\sigma_{h 1}^{2} & \sigma_{h 1 h 2}^{2} \cdots \cdots \cdots & \sigma_{h 1 h u}^{2} \\
& \sigma_{h 2}^{2} \cdots \cdots \cdots \cdot & \sigma_{h 2 h u}^{2} \\
\cdots \cdots & \cdots \cdots & \cdots \cdots & \cdots \cdots \\
& & & \sigma_{h u}^{2}
\end{array}\right)
$$

The values $\sigma_{\mathrm{hi}}^{2}$ are compared with the adopted ones, which are computed from the criterion matrix ( say $\sigma_{\text {hio }}^{2}$ ), then find the ratios :

$$
r_{i}=\frac{\sigma_{h_{1}}^{2}}{\sigma_{h_{10}}^{2}}
$$

Then, the weight vector $\left(p_{1}\right)$ is multiplied by the largest value ( $\left.\mathrm{ri}_{\max }\right)$, and the resulted weight vector will be $\left(p_{2}\right)$. After that, the new covariance matrix is computed. Also, if the difference between the ratios is large, 
the use of the value $\left(\dot{r}_{\max }\right)$ will lead to some variances to be more smaller and give a precision more greater than the required, and consequently to high cost without any need. Here, an iterative modification to the weight vector $p_{1}$ using the necessary observations, which affect the weak parts of the network, can be done by increasing the weights of these observations or the repetition numbers of them.

The choice of the method of the modification, will depend on the preliminary solution vector, and the criteria reproduced by substituting by this vector. Firstly, the actual repetition numbers according to the preliminary solution $\left(p_{1}\right)$ are evaluated. If it does not satisfy the required criteria, the modification is done using any of the methods mentioned above.

\section{CASE-STUdIES}

In this investigation, some case-study, used as sample examples, will be solved, using the simple least least-squares solution, approximating an inverse criterion matrix. The selected case-studies, in our case here, will be concentrated on two problems, namely : the geodetic networks in onedimensional and two dimensional networks. The first deals with the levelling networks, while the second deals with the horizontal twodimensional networks. Also, it is to be mentioned that, in the case-studies, only, the random errors are considered, assuming that the gross errors and the systematic errors are eliminated a priori. Thus, the realization of the results, will depend on the random errors only .

\subsection{THE ADOPTED CRITERIA}

In the case-studies, the precision criteria formed as a criterion matrix will be considered. In the case-studies, the measures of precision will be : the absolute error ellipses in the two dimensional networks, while in levelling networks, the precision criterion will be the standard deviations of the heights of the points. Thus, the formulation of the criterion matrices, and the analysis of the results, will depend on these criteria.

Recall that, the network is well designed, if the error situation fulfils the properties of homogeneity and isotropy. In the case studies, the criterion matrices will ensure these properties. This means that, the postulated absolute error ellipses all over the network will be circles with equal radius. For the levelling networks, all the standard deviations of the stations will be equally sized. The most suitable configuration which ensures the properties of homogeneity and isotropy, is the free network. Thus, the error propagation will be more homogeneous in this type of networks. This is because, the free network adjustment can be imagined as, a constrained adjustment with fixing the center of gravity of the network. The concept of the free network is also suitable for the general purpose network, where the properties of homogeneity and isotropy are required. 


\subsubsection{THE CRITERION MATRIX CONSIDERING THE ABSOLUTE ERRORS ELLIPSES}

The most practical choice of the criterion matrix here is the unite matrix. This is suitable for all networks, which have these properties of homogeneity and isotropy. The unit matrix will be considered as the designated cofactor matrix, and if the requirement of the net is any scalar of variances, this scaler value will be expressed as the unit variance $\sigma_{0}^{2}$. The solution of the SOD problem will be due to the unit matrix, and the final results (the realization) will be obtained using $\sigma_{0}^{2}$.The unit matrix will be denoted by $Q_{\mathbf{I}}$ in the text.

\subsubsection{THE CRITERION MATRIX FOR LEVELLING NETWORKS}

Here, the standard deviations of heights are required to be equally sized. So, the most suitable cofactor matrix, which fulfils this property is the unit matrix also, $\mathbf{Q}_{\mathbf{I}}$. The unit matrix will be used as cofactor matrix, when solving the example of the levelling network

\section{PRESENTATION AND DISCUSSION OF THE OBTAINED RESULTS FROM THE SOLUTION OF SELECTED CASE- STUDIES}

The second order design problem will be solved for two case-studies. One of them will be for levelling network, and the other for a triangulation network. All the case-studies will be solved for the free network concept . The criterion cofactor matrix, in all cases, will be the unit matrix, which ensures the properties of homogeneity and isotropy. This criterion is the suitable one, for the general purpose networks. The realization of the results will be performed for the two case-studies. This realization will be according to a selected actual criterion covariance matrix, and selected type of instruments, which give specific precision of the measured quantities. Firstly, the solution weight vector will be obtained according to the SOD with respect to the cofactor matrix $Q_{1}$, then, the realization will be done using the constant term $\sigma_{0}^{2}$.

\subsection{CASE-STUDY NO.(1) : A LEVELLING NETWORKS}

A levelling network is shown in Fig.(1). It consists of 7 bench-marks, to be determined from 10 levelled height differences. The desctiption of the measured height differences, is shown in Table (1). Firstly, the unite matrix $Q_{1}$, is transformed to the free net datum, by the relation

$$
Q_{\text {lred }}=A^{+} A Q_{I} A^{+} A
$$

The preliminary weight vector $p_{1}$ involves positive values only (Table 1 ). The standard deviations resulted from $p_{1}$ are ranged from 1.06 at points $\mathrm{B}, \mathrm{E}, \mathrm{F}$ to 1.26 at $\mathrm{A}$ (Table 2 ). The modification of the vector $\mathrm{p}_{1}$ will depend on the point $A$, and the factor of modification will equal (1.26 )$^{2}=1.59$. The modified weight vector $\mathrm{p}_{2}$ is also shown in Table (1). The reproduced criteria ( standard deviations) according to the vector $\mathrm{p}_{2}$, will be 
ranged from 0.83 at points $B, E$ to 1.00 at point $A$. The realization of the results will be done using the vector $\mathrm{p}_{1}$. The actual covariance matrix ensures that the standard deviations of the heights at all points will equal $1.00 \mathrm{~mm}$. It is assumed that, it is possible to level to a precision equal $1.0 \mathrm{Vd}$ $\mathrm{mm}$, where $\mathrm{d}$ is the levelled distance in $\mathrm{km}$. Table (1) shows the realization of the results. For each height difference, it shows, the variance of a single levelling, the required variance, and the required repetition numbers. The repetition numbers are approximated to the largest integer value. The actual criteria ( the standard deviations of heights) are also computed in $\mathrm{mm}$ in Table (2). The resulted repetition numbers $\left(n_{1}\right)$ lead to that, the required criteria are fulfilled at all points except for point $A$, which has a standard deviation of height equals $1.16 \mathrm{~mm}$. So, the repetition numbers of the observations, which affect $A$ ( the levelled lines A-B and A-E) are increased by one. The resulting repetition numbers $\left(n_{2}\right)$ are used to compute the new criteria. The resulting standard deviation of the height at $A$ will be 1.06. It can be improved by the same procedure.

TABLE 1 The preliminary weight vector $\left(p_{1}\right)$, the modified one $\left(p_{2}\right)$, and the realization of the results using $\mathrm{p}_{1}$, for the SOD of the levelling network. The variance of a single levelling is assumed to be $1.00 * \mathrm{~d} \mathrm{~mm}$, where $\mathrm{d}$ is the distance in $\mathrm{km}$. All the standard deviations of the heights are required to be $1.00 \mathrm{~mm}$.

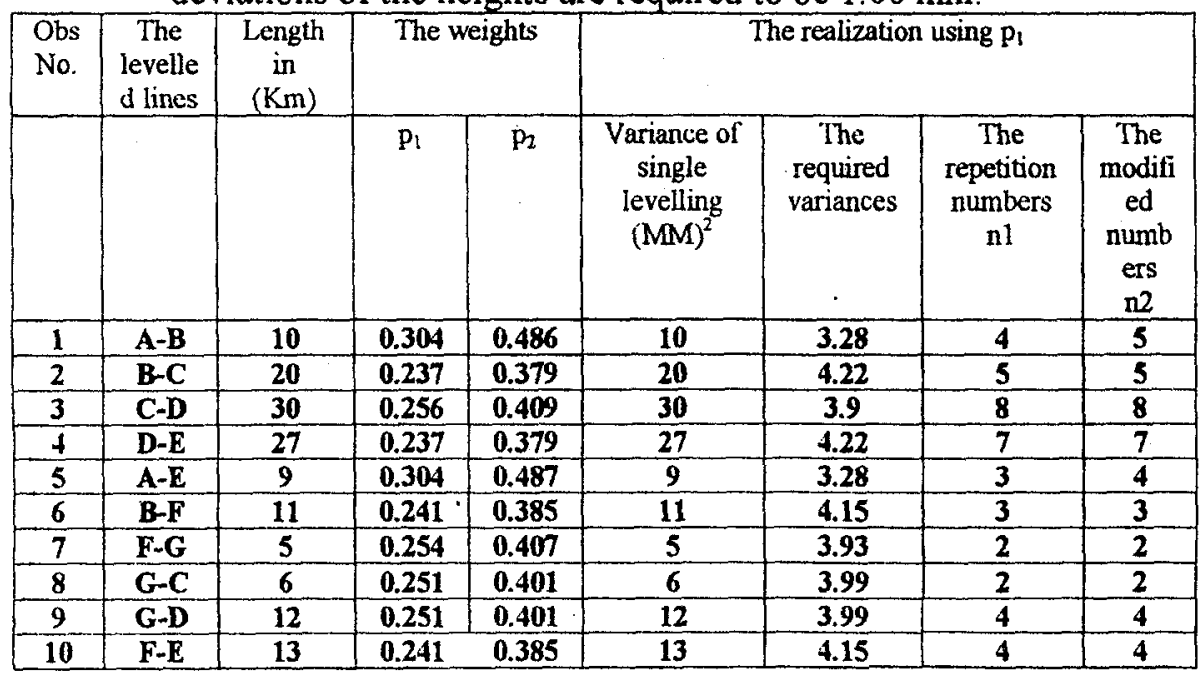

TABLE 2. The reproduced standard deviations of the heights of the points in the levelling network

\begin{tabular}{|c|c|c|c|c|}
\hline \multirow[t]{2}{*}{ Points } & \multicolumn{2}{|c|}{$\begin{array}{l}\text { Fom the design according to the } \\
\text { cofactor matrix } Q_{1}\end{array}$} & \multicolumn{2}{|c|}{$\begin{array}{l}\text { From the realization } \\
\text { according to } p_{1}\end{array}$} \\
\hline & $\begin{array}{l}\text { From } \mathbf{p}_{1} \\
\text { (unitless) }\end{array}$ & $\begin{array}{c}\text { From p } \\
\text { (unitless) }\end{array}$ & $\begin{array}{l}\text { due to } \mathbf{n}_{1} \\
\text { (mm) }\end{array}$ & $\begin{array}{l}\text { due to } n_{2} \\
\text { (mm) }\end{array}$ \\
\hline $\mathbf{A}$ & 1.26 & 1.00 & 1.16 & 1.06 \\
\hline B & 1.06 & 0.83 & 0.98 & 0.94 \\
\hline C & 1.09 & 0.87 & 1.02 & 1.02 \\
\hline D & 1.09 & 0.87 & 1.02 & 1.01 \\
\hline $\mathbf{E}$ & 1.06 & 0.83 & 0.97 & 0.95 \\
\hline $\mathbf{F}$ & 1.06 & 0.84 & 0.92 & 0.92 \\
\hline G & 1.10 & 0.87 & 0.94 & 0.94 \\
\hline
\end{tabular}




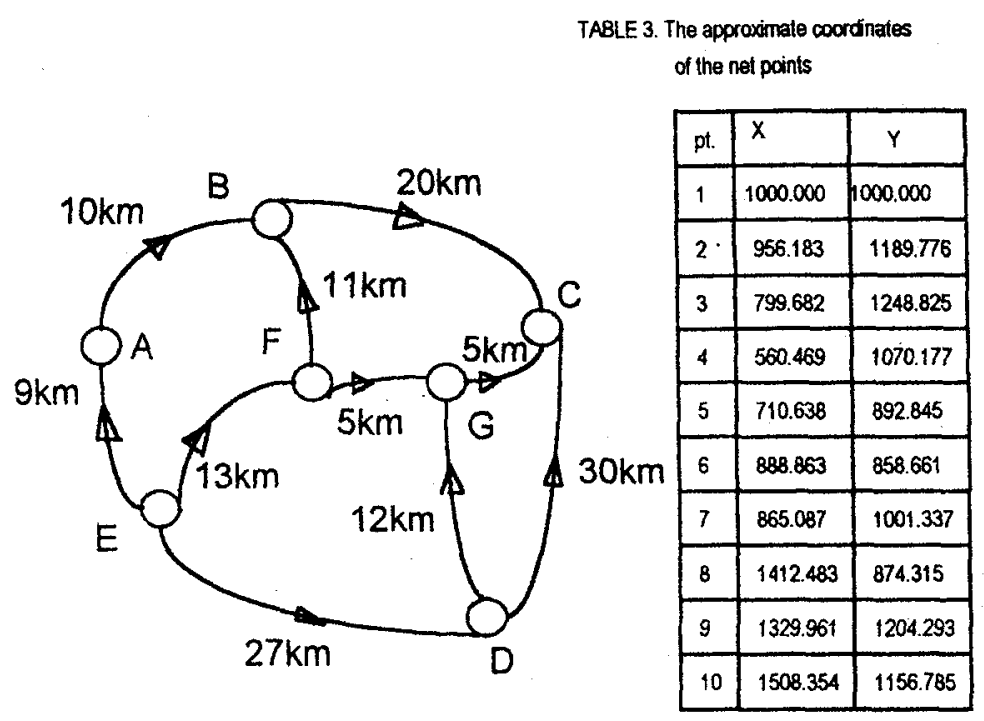

FIG. 1 A levelling Network

\subsection{CASE-STUDY NO.2 : A triangulation network}

Figure (2) shows a free triangulation network, with its approximate coordinates. This network consists of 10 points ( 20 coordinates), and the observational plan includes the angles, described in Table ( 4 ). The cofactor criterion matrix is the unit matrix $\left(Q_{1}\right)$. Firstly, the criterion matrix is transformed to the free net datum. Then, the SOD solution is obtained, which is the weight vector $p_{1}$ (Table 4 ). All the resulting weights are positive, except for the observation no.(13), which is the angle 4-5-7, with negative value. This observation is discarded, and the solution vector $p_{1}$ is recomputed. All the resulting weights are positive. The elements of the error ellipses are determined ( Table 5 ). The semi-major axes are ranged from 1.29 at points 7,9 to 2.98 at point 6 . The preliminary weight vector is modified to $\mathrm{p}_{2}$. The scaler of modification is the square of $(2.98)$. The criteria are recomputed ( Table 5 ). The semi-major axes are now ranged from 1.00 at point 6 to 0.43 at points 7,9 . Figure (3) shows the error situation reproduced by the modified weight vector $p_{2}$

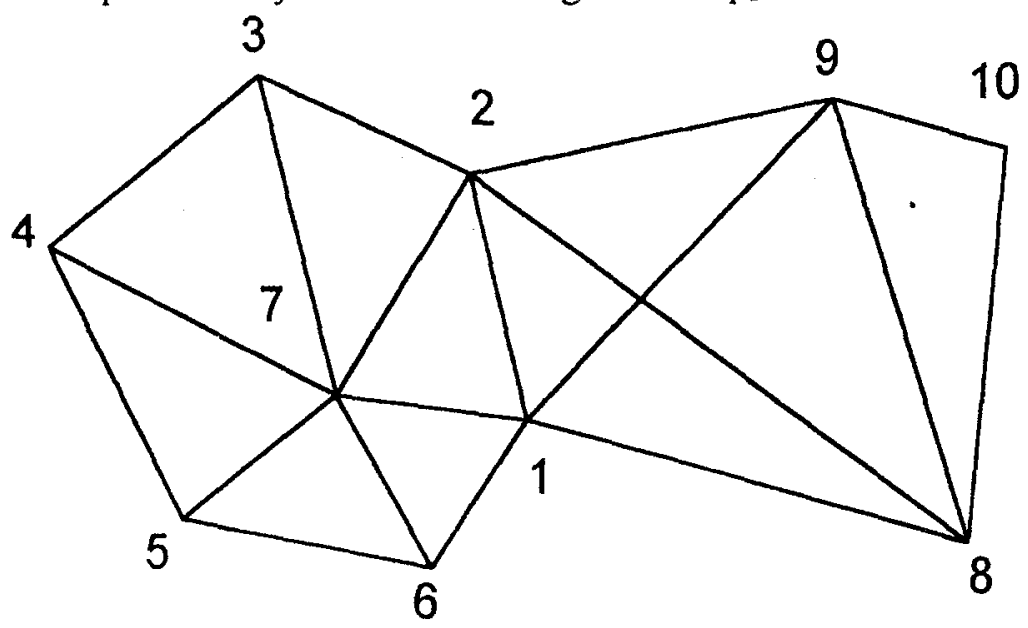

FIG. 2 A triangulation network 
TABLE 4. The preliminary weight vector $p_{1}$ ( resulting after one discarding step), and the modified vector $\mathrm{p}_{2}$, resulting from the SOD of the triangulation network.

\begin{tabular}{|c|c|c|c|c|}
\hline $\begin{array}{l}\text { Obs. } \\
\text { No. }\end{array}$ & $\begin{array}{l}\text { Description } \\
\text { of the }\end{array}$ & \multicolumn{2}{|c|}{$\begin{array}{l}\text { The preliminary solution } \\
\text { vector } \mathrm{p}_{1}\end{array}$} & $\begin{array}{c}\text { The } \\
\text { modified }\end{array}$ \\
\hline$I$ & $9-1-8$ & $0.7192 * 10^{-6}$ & $0.7207 * 10^{-6}$ & $0.6402^{*} 10^{-5}$ \\
\hline 2 & $2-1-9$ & $0.0971^{*} 10^{-6}$ & $0.0983 * 10^{-6}$ & $0.0873^{*} 10^{-5}$ \\
\hline 3 & $7-1-2$ & $0.0272 * 10^{-6}$ & $0.0260 * 10^{-6}$ & $0.0231 * 10^{-5}$ \\
\hline 4 & $6-1-7$ & $0.0490^{*} 10^{-6}$ & $0.0475^{*} 10^{-6}$ & $0.0422 * 10^{-5}$ \\
\hline 5 & $9-2-8$ & $0.5573 * 10^{-6}$ & $0.5569^{*} 10^{-6}$ & $0.4947 * 10^{-5}$ \\
\hline 6 & $8-2-1$ & $0.1553 * 10^{-6}$ & $0.1565^{*} 10^{6}$ & $0.1390^{*} 10^{-5}$ \\
\hline 7 & $1-2-7$ & $0.0803^{*} 10^{-6}$ & $0.0766^{*} 10^{6}$ & $0.0680^{*} 10^{-5}$ \\
\hline 8 & $7-2-3$ & $0.0120^{*} 10^{-6}$ & $0.0101 * 10^{-6}$ & $0.0090 * 10^{-5}$ \\
\hline 9 & $2-3-7$ & $0.1815^{*} 10^{-6}$ & $0.1837 * 10^{-6}$ & $0.1632^{*} 10^{-5}$ \\
\hline 10 & $7-3-4$ & $0.2234 * 10^{-6}$ & $0.2213 * 10^{-6}$ & $0.1966^{*} 10^{-5}$ \\
\hline 11 & $3-4-7$ & $0.4937^{*} 10^{-6}$ & $0.4966^{*} 10^{-6}$ & $0.4411 * 10^{-5}$ \\
\hline 12 & $7-4-5$ & $0.5393^{*} 10^{-6}$ & $0.5011^{*} 10^{6}$ & $0.4451 * 10^{-5}$ \\
\hline 13 & $4-5-7$ & $-0.0315^{*} 10^{-6}$ & & \\
\hline 14 & $7-5-6$ & $0.1560 * 10^{-6}$ & $0.1591 * 10^{-6}$ & $0.1413 * 10^{-5}$ \\
\hline 15 & $5-6-7$ & $0.0621^{*} 10^{-6}$ & $0.0643^{*} 10^{-6}$ & $0.0571^{*} 10^{-5}$ \\
\hline 16 & $7-6-1$ & $0.0792 * 10^{-6}$ & $0.0831 * 10^{-6}$ & $0.0739 * 10^{-5}$ \\
\hline 17 & $2-7-1$ & $0.0564 * 10^{-6}$ & $0.0596 * 10^{-6}$ & $0.0530^{*} 10^{-5}$ \\
\hline 18 & $3-7-2$ & $0.3318^{*} 10^{-6}$ & $0.3328 * 10^{-6}$ & $0.2956 * 10^{-5}$ \\
\hline 19 & $4-7-3$ & $0.3067^{*} 10^{-6}$ & $0.3067 * 10^{-6}$ & $0.2724 * 10^{-5}$ \\
\hline 20 & $5-7-4$ & $0.3727 * 10^{-6}$ & $0.3367 * 10^{-6}$ & $0.2991 * 10^{-5}$ \\
\hline 21 & $6-7-5$ & $0.0678 * 10^{-6}$ & $0.0637 * 10^{-6}$ & $0.0566 * 10^{-5}$ \\
\hline 22 & $1-7-6$ & $0.0118 * 10^{-6}$ & $0.0106 * 10^{-6}$ & $0.0094^{*} 10^{-5}$ \\
\hline 23 & $9-8-10$ & $0.8492 * 10^{-6}$ & $0.8493^{*} 10^{-6}$ & $0.7543^{*} 10^{-5}$ \\
\hline 24 & $2-8-9$ & $0.8080^{*} 10^{-6}$ & $0.8115^{*} 10^{-6}$ & $0.7208^{*} 10^{-5}$ \\
\hline 25 & $1-8-2$ & $0.7997 * 10^{-6}$ & $0.8038^{*}+10^{6}$ & $0.7140^{*} 10^{-5}$ \\
\hline 26 & $10-9-8$ & $0.3202 * 10^{-6}$ & $0.3199 * 10^{-6}$ & $0.2842 * 10^{-5}$ \\
\hline 27 & $8-9-1$ & $0.3473 * 10^{-6}$ & $0.3453 * 10^{-6}$ & $0.3067 * 10^{-5}$ \\
\hline 28 & $1-9-2$ & $0.4804 * 10^{-6}$ & $0.4704^{*} 10^{-6}$ & $0.4178 * 10^{-5}$ \\
\hline 29 & $8-10-9$ & $0.0333 * 10^{-6}$ & $0.0334^{*} 10^{-6}$ & $0.0297 * 10^{-5}$ \\
\hline
\end{tabular}

TABLE 5.The resulting criteria, from the SOD solution, of the triangulation network, by the preliminary vector $p_{1}$ and by the modified vector $p_{2}$.

\begin{tabular}{|c|c|c|c|c|c|c|c|c|c|c|}
\hline Pt & \multicolumn{4}{|c|}{$\begin{array}{c}\text { The criteria reproduced by the } \\
\text { preliminary weight vector }\end{array}$} & \multicolumn{4}{c|}{ The criteria reproduced by the modified } \\
& \multicolumn{4}{|c|}{ weight vector $p_{2}$} \\
\hline & $\sigma_{\mathrm{x}}$ & $\sigma_{\mathrm{y}}$ & $\mathrm{a}$ & $\mathrm{b}$ & $\phi$ & $\sigma_{\mathrm{x}}$ & $\sigma_{y}$ & $\mathrm{a}$ & $\mathrm{b}$ & $\phi$ \\
\hline 1 & 2.25 & 2.02 & 2.27 & 2.00 & 9.47 & 0.76 & 0.68 & 0.76 & 0.67 & 9.45 \\
\hline 2 & 2.09 & 2.45 & 2.45 & 2.08 & -3.85 & 0.7 & 0.82 & 0.82 & 0.70 & -3.85 \\
\hline 3 & 2.45 & 2.42 & 2.51 & 2.35 & 34.31 & 0.82 & 0.81 & 0.84 & 0.79 & 34.31 \\
\hline 4 & 2.7 & 2.77 & 2.79 & 2.68 & 13.97 & 0.9 & 0.93 & 0.93 & 0.90 & 13.97 \\
\hline 5 & 2.26 & 2.15 & 2.31 & 2.11 & 18.76 & 0.76 & 0.72 & 0.77 & 0.71 & 18.77 \\
\hline 6 & 2.95 & 2.76 & 2.98 & 2.74 & -10.9 & 0.99 & 0.93 & 1.00 & 0.92 & -10.9 \\
\hline 7 & 1.26 & 1.25 & 1.29 & 1.22 & -32.5 & 0.42 & 0.42 & 0.43 & 0.41 & -32.5 \\
\hline 8 & 2.23 & 2.32 & 2.33 & 2.22 & -11.6 & 0.75 & 0.78 & 0.78 & 0.75 & -11.6 \\
\hline 9 & 1.23 & 1.21 & 1.29 & 1.15 & 36.2 & 0.41 & 0.41 & 0.43 & 0.39 & 36.2 \\
\hline 10 & 2.00 & 2.12 & 2.12 & 2.00 & 4.34 & 0.67 & 0.71 & 0.71 & 0.67 & 4.34 \\
\hline
\end{tabular}




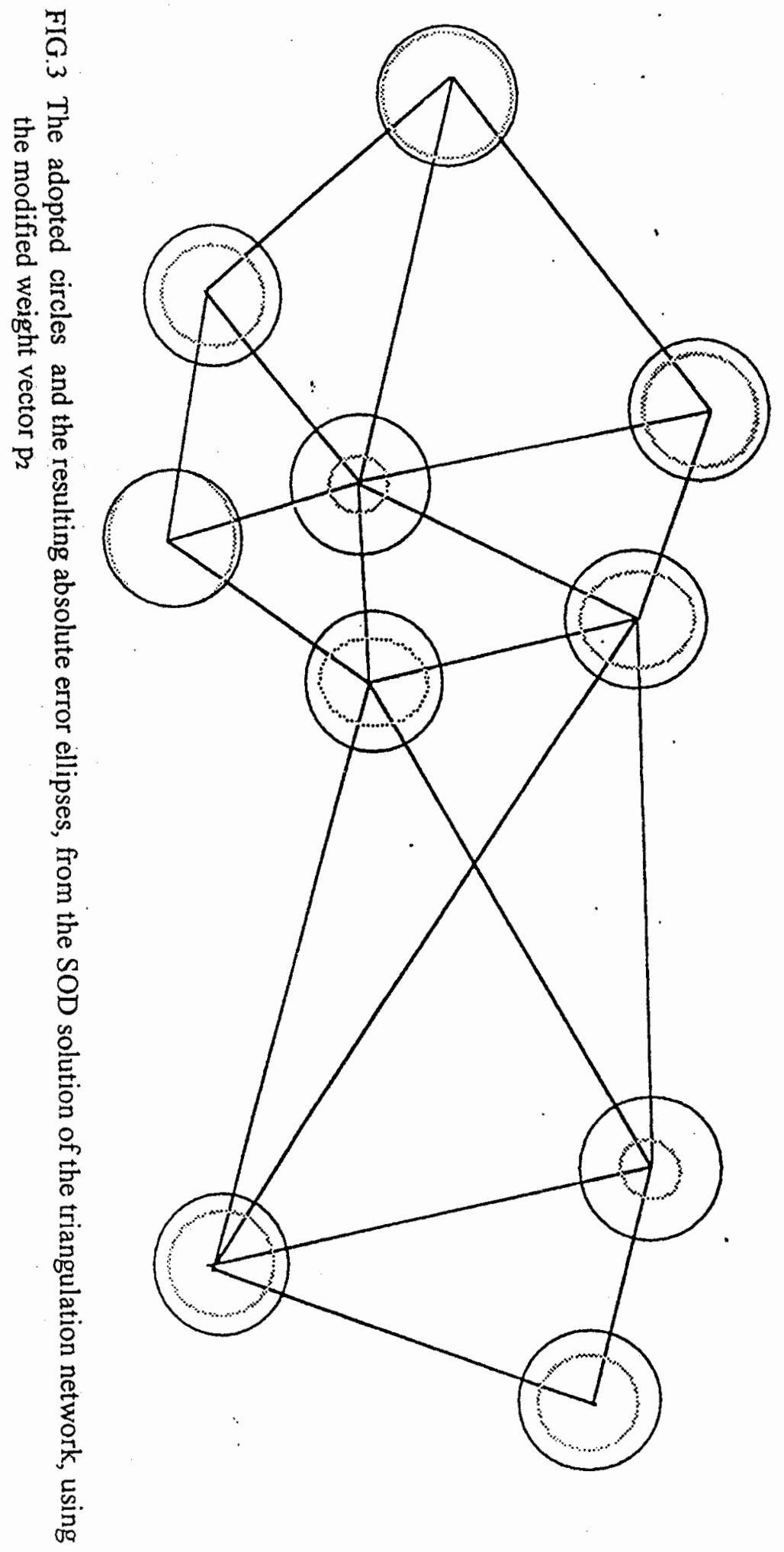


$\sigma_{\mathrm{x}}=$ the standard deviation of the $\mathrm{x}$-coordinates

$\sigma_{y}=$ the standard deviation of the $y$-coordinates

$a=$ the semi-major axis of the error ellipse

$b=$ the semi-minor axis of the error ellipse

$\phi=$ the oriention of the semi-major axis with respect to the $y$-axis

\section{CONCLUSIONS}

One of the main problems which may arise when solving the SOD ; problem, is that, the reslting weight vector may not reproduce a cofactor matrix, which ensure the criteria requirements, in some parts of the network; or all over the network. This problem is treated by modifying the resulting weight vector linearly, by its multiplication by a scaler value. This scaler is determined from the comparison between the resulted criteria and the postulated one .

According to the results of this study, the following conclusions can be drawn:

- The results of the solution of the second-order design problem will be the optimal weights, which must be assigned to the observations, to satisfy the required criteria under minimum cost ( or a specific cost). From these weights, the required variances of the observations are evaluated. This leads to a detailed design of the required instruments, procedures and repetition numbers which yield the required variances (or weights).

- If the resulting weight vector does not satisfy the required criteria (or some of them), it can be linearly modified, by its multiplication by a suitable scalar. This scalar is determined depending on the comparison, between the resulting criteria, and the adopted one such that the resulting criteria- at least-equal the adopted one.

The choice ofthe method of the modification, will depend on thr preliminary solution vector, and the criteria reproduced by substituting by this vector. Firstly, the actual repetition numbers according to the preliminary solution ( $\left.p_{1}\right)$ are evaluated. If it does not satisfy the required criteria, the modification is done using any of the proposed methods.

-The presented examples show the visibilty of the alternative approach for the solution of the SOD problem.

-The solution of the examples has proved that, the alternative approach, is considerably more simple and economic than using the conventional technique.

- The mostly used criterion matrices, for the general purpose network, are those give the properties of homogeneity and isotropy. The Taylor-Karmen Structured criterion matrices have great success for these properties. The unit matrix can be considered as a special case of these matrices, where zero correlation functions are imposed for the longitudinal and transversal errors.

\section{REFERENCES}

Ahmed F.A.G. (1999): “Numerical efficient solutions of the second-order design ( the optimal weights) of geodetic networks".M.Sc. thesis, aculty of Engineering, Shebin El-Kom, Minufiya University, Egypt. 
Assal,F.F.(1994):“ Deformation measurements and and analysis of movements in geodetic networks". M.Sc.Thesis, Faculty of Engineering, Shebin El-Kom, Minufiya University, Egypt.

Grafarend, E. and Schaffrin,B. (1979): "Kriterion-Matrizen I " ZFV 104,133-149.

Hassouna,R.(1993): “Application of free network adjustment in horizontal and verical control "M.Sc.Thesis, Faculty of Engineering, Shebin El-Kom, Minufiya University, Egypt.

Kuang,S.(1993): "Second order design: Shooting for maximum reliability " . Journal of Surveying Engineering, Vol. 119,3, PP. 102-110.

Nassar, M.; Shaker, A. and Assal, F. (1994) : Successivesolutions for diffedent orders of the optimal design problems for local horizontal geodetic monitoring networks, using simulation process " Proceedings of the 2 nd Conference on Civil Engineering, Military Technical Collage, 8-10 Nov. 1994.

Nassar,M.; and Mousa, A. (1992a) : " Measures of quality as applied to crustal movement monitoring geodeticnetworks ".Proceedings of the Fourth Scientific General Meeting,National Research Institute of Astronomy and Geophysics, Helwan, Cairo, Nov. 2325,1992 .

Nassar,M; and Mousa, A. (1992b) : "Outlier detection in geodetic networks ". Proceedings of the Fourth Scientific General Meeting, National Research Institute of Astronomy and Geophysics, Helwan, Cairo, Nov. 23-25,1992.

Schaffrin, B. (1981a): Some considerations on the optimal design of geodetic networks. paper presened to : IAG Symp. on Geodetic Networks and Computations, Munchen

Schaffrin, B. (1981b) :" Some proposal concerning the diagonal second order design of geodetic networks " Manuscripta Geodetica,6(3),303-326

Schmitt,G. (1978): :Numerical problems concerning the second order design of geodetic networks". Proceeding second international symposium on problems related to the redifination of north geodetic networks, Arlington 1978, 555-565.

Schmitt,G. (1979):" Experiences with the second order design problem' in theoretical and practical geodetic networks". Proceeding international symposium on optimization of design and computation of control networks. Edited by Hamus, Sopron, Hungary.

Von Gelder, B.H., 1994, " Geodetic Control Survey: Lecture Notes ", Purdue University, Civil Eng. Dep., West Lafayette, IN., USA. 
حل بديل أكثر بساطة لمسألة التصميم اللانية لشبكات المليلات الجيرديسية

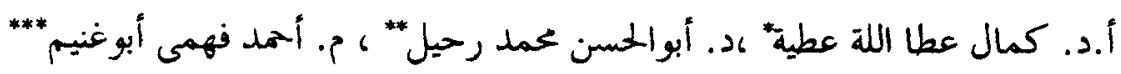

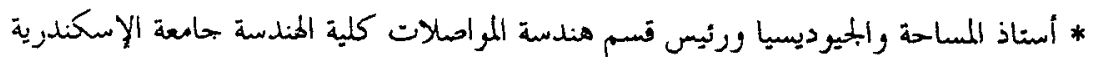

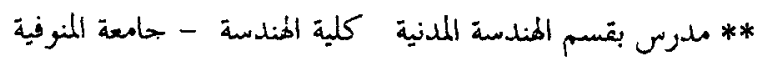
م مهندس مدلن تصميم التبكات البحيوديسية يعنى الإختيار الأمثل لمستوى المقارنة أو نظـــــام إحلداثيـــات

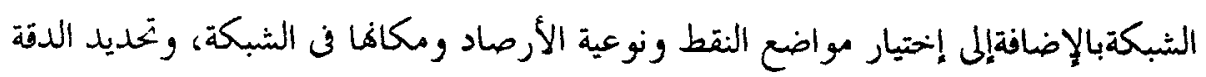

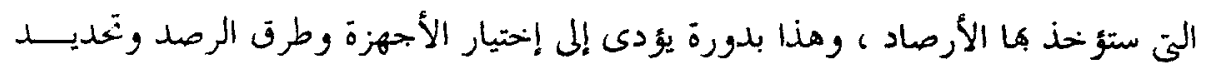

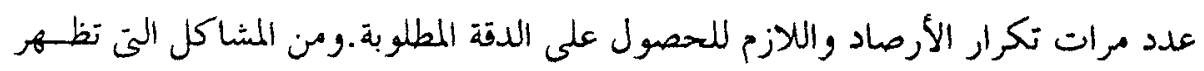

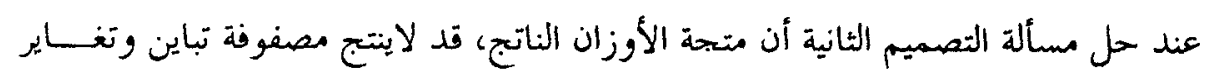

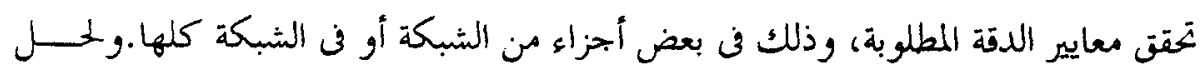

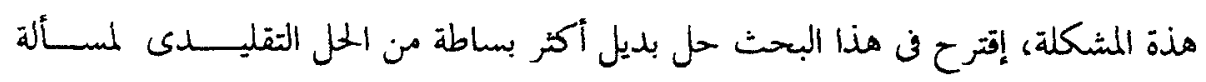

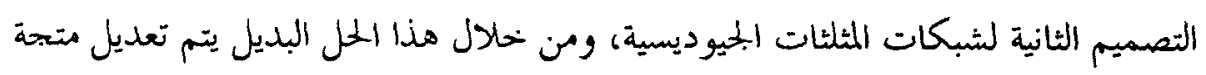

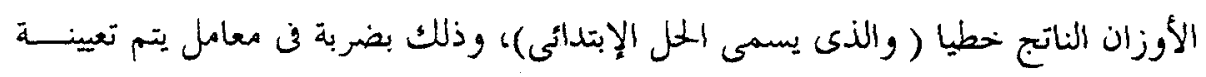

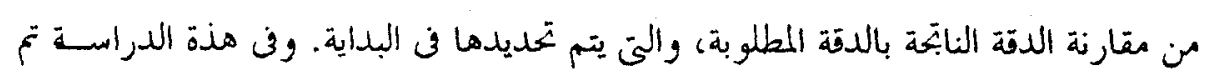

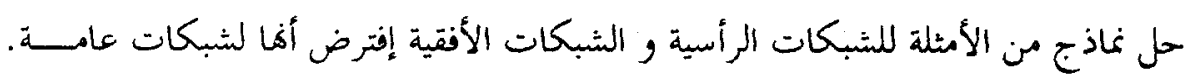

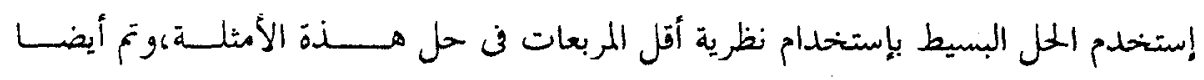

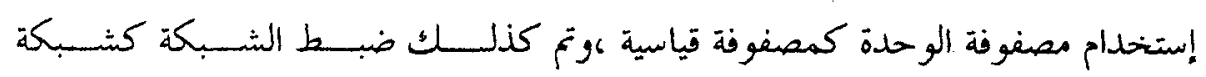

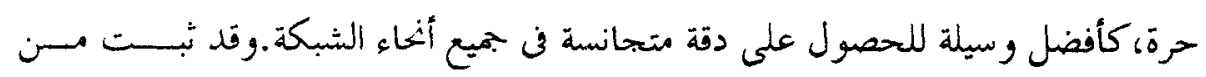

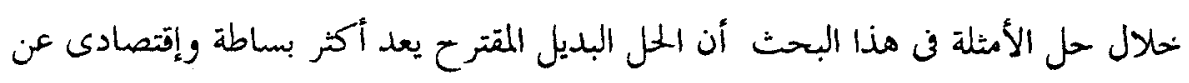

الطريقة التقليدية ـ وقد تع من خلال هذا البحث التوصل إلى عدد من التوصيات منها:

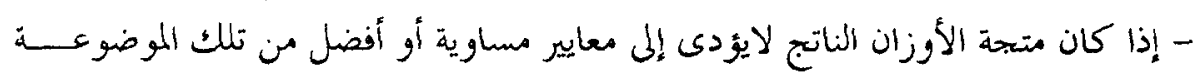

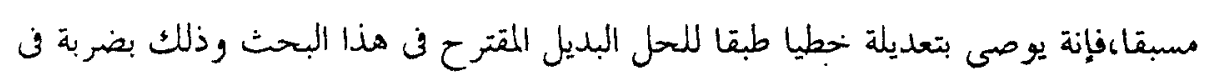

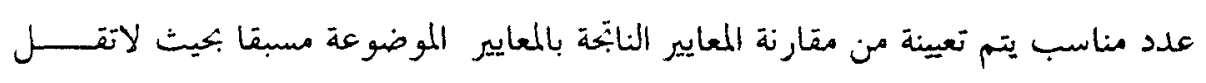

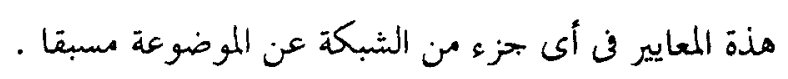

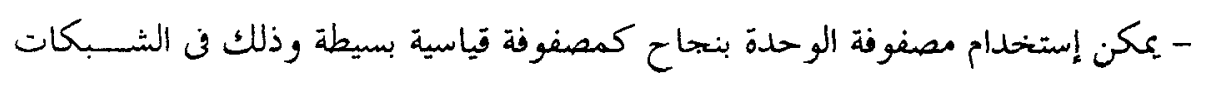

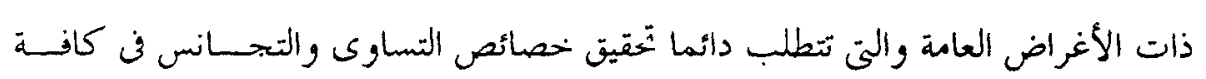

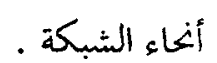

